\title{
FACTORES MO'TIVACIONALES QUE INCIDEN EN EL APRENDIZAJE DE FÍSICA
}

\section{Incident motivational factors in Physics learning}

\author{
Chess Emmanuel Briceño Núñez \\ ORCID: https://orcid.org/0000-0002-1712-4136 \\ Unidad Educativa Internacional Bilingüe Giuseppe Garibaldi, Guayaquil-Guayas, Ecuador. \\ chesspiare@gmail.com
}

\section{Resumen:}

El aprendizaje de física es un asunto trascendental en el proceso formativo de los estudiantes de Bachillerato General Unificado (BGU), es por ello que es una asignatura indispensable y obligatoria en el sistema educativo ecuatoriano. La presente investigación tiene como objetivo identificar el grado de motivación así como los factores motivacionales presentes en el aprendizaje de física. Por su naturaleza este estudio es cuantitativo, de tipo descriptivo, con un diseño no experimental transversal. Se llevó a cabo un muestro intencional en el que se seleccionó a 330 estudiantes activos de Bachillerato General Unificado (BGU) de un colegio privado de Guayaquil, Ecuador. Se aplicó un test contentivo de 32 preposiciones afirmativas presentadas en una escala Likert de 5 puntos. Los factores considerados fueron: el interés de la física como asignatura, la intensidad de la motivación del estudiante, la actitud hacia el docente de física, la actitud hacia el aprendizaje de física, las ventajas a futuro que da el saber física, el deseo de aprender física y la actitud hacia el entorno escolar y los compañeros con los que se aprende física. Los resultados indican que los estudiantes se encuentran altamente motivados hacia el aprendizaje de física y que los estudiantes de tercer año de BGU son quienes presentan mayor grado de motivación. Igualmente los datos indican que los hombres tienen un mayor nivel de motivación.
\end{abstract}

Palabras clave: Aprendizaje, estudiante de bachillerato, estudiante de secundaria, física, motivación.

\begin{abstract}
:
The learning of physics is a transcendental matter in the formative process of the students of the Unified General Baccalaureate (BGU), which is why it is an indispensable and compulsory subject in the Ecuadorian educational system. The present research aims to identify the degree of motivation as well as the motivational factors present in the learning of physics. By its nature, this study is quantitative, descriptive, with a non-experimental cross-sectional design. An intentional sampling was carried out in which 330 active students of the Unified General Baccalaureate (BGU) from a private school in Guayaquil, Ecuador were selected. A test of 32 affirmative prepositions presented on a 5-point Likert scale was applied. The factors considered were: the interest in physics as a subject, the intensity of the student's motivation, the attitude towards the physics teacher, the attitude towards the learning of physics, the future advantages of physical knowledge, the desire to learning physics and the attitude towards the school environment and peers with whom one learns physics. The results indicate that students are highly motivated towards learning physics and that BGU thirdyear students are the ones who show the highest degree of motivation. Likewise, the data indicate that men have a higher level of motivation.
\end{abstract}

Keywords: Learning, high school student, Physics, motivation. 


\section{Introducción}

Desde temprano las instituciones educativas deben enfatizar la importancia de estudiar física, ya que por sí mismos, los estudiantes pueden no apreciar completamente las ventajas de la física en la vida diaria. La física es uno de los campos del conocimiento que subyace en el universo físico y se aplica constantemente a la vida cotidiana de las personas. Las ventajas de la física en la vida cotidiana pueden ser tan básicas como la conversión de energía eléctrica en calor para hacer calentar algún alimento o tan complejas como trazar el vuelo de un transbordador espacial desde nuestro planeta Tierra a la órbita. Aunque algunos conceptos son inicialmente complejos de comprender, se debe ayudar a los estudiantes a que comprendan que la recompensa en términos de satisfacción y conocimiento puede hacer que ese esfuerzo valga la pena dada la incalculable importancia de la física en la ciencia.

Es importante que se ayude a los estudiantes a que en un nivel individual y personal comprendan que estudiar física puede ser gratificante en la medida en la que ellos comienzan a comprender los objetos y sucesos cotidianos en términos de los conceptos de física que los subyacen, como la aceleración de un automóvil, la gravedad que hace que una manzana caiga del árbol o la energía eléctrica. Cuando un estudiante comprende plenamente que la física no es un campo de estudio aislado o independiente, comienza a descubrir que existe una amplia gama de campos para sus estudios universitarios en el futuro. Por ejemplo, los estudiantes de medicina deben conocer algo de física básica para comprender cómo fluye la sangre y el aire en el cuerpo, teniendo en cuenta conceptos como la presión, la velocidad del flujo y los cambios en la resistencia al flujo. La física también es parte integral de la ingeniería y, en general, es relevante para todas las ciencias.

- Algunos estudiantes se sienten ansiosos al tener que estudiar física, ya que se ha creado una reputación de ser una asignatura difícil. Otros no comprenden la importancia de estudiar física o las ventajas de la física en la vida cotidiana. Los aspectos específicos de la física que muchas personas encuentran abrumadores incluyen la necesidad de comprender diferentes ecuaciones matemáticas y gráficos y luego poder traducir esos conceptos a la vida real. Es cierto que la dificultad en el aprendizaje se debe a un asunto bastante subjetivo que obedece a un cúmulo de elementos circunstanciales e individuales, pero es igualmente cierto que uno de los valores de estudiar física es que es intrínsecamente desafiante $y$, por lo tanto, también extremadamente gratificante cuando un estudiante lo entiende. Por todo lo antes expuesto entonces compete al estudio que a continuación se presenta el estudio de los factores motivacionales que inciden en el aprendizaje de física, teniendo como objetivos precisamente el identificar los niveles motivacionales de los estudiantes de BGU que participan como muestra así como el grado de incidencia de cada uno de los factores que se consideran en esta investigación.

Resulta conveniente revisar algunas investigaciones vinculantes al estudio que se presenta, como la de Luque (2017) que en su tesis doctoral aborda los elementos relacionados a los estilos de aprendizaje y su relación con el rendimiento escolar en física, de los estudiantes del quinto de secundaria en la Institución Educativa $\mathrm{N}^{\circ} 17$, V.E.S. - 2016. La investigación se realizó bajo el enfoque cuantitativo y el método hipotético deductivo, del tipo básica, de diseño no experimental, correlacional y de corte transeccional. La muestra estuvo constituida por 100 estudiantes y para determinar sus 
estilos de aprendizaje se utilizó como instrumento el cuestionario de Honey Alonso (CHAEA-1992), la validez se llevó a cabo por juicio de expertos y para la confiabilidad se tomó una prueba piloto a 21 estudiantes, obteniendo un coeficiente de confiabilidad Kuder Richardson de 0.875, así mismo, se obtuvo datos sobre el rendimiento escolar del registro oficial de notas correspondiente al tercer bimestre del 2016.

De acuerdo a los resultados de la estadística inferencial se llegó a la conclusión que existe una buena correlación, positiva y estadísticamente significativa entre los estilos de aprendizaje, reflexivo, teórico y el rendimiento escolar en física de los estudiantes del quinto de secundaria, con un Rho de Spearman de 0.624 y 0.727 respectivamente. Atendiendo a la forma tradicional de impartir las clases de física, empleando un enfoque marcadamente teórico. Así mismo se encontró una correlación baja y negativa entre el estilo activo y el rendimiento escolar en física ya que se obtuvo un Rho de Spearman de -0.336, con un p-valor de 0.001 en los tres casos, lo que demuestra una correlación inversa, en la que los estudiantes con estilo activo se caracterizan por su dinamicidad, cuando estas conductas aumentan, presentan condiciones desfavorables para un buen desempeño escolar. Por otro lado no se pudo demostrar la relación entre la dimensión pragmática de los estilos de aprendizaje y el rendimiento escolar en física, dado que se obtuvo un p-valor de 0.357.

Briceño et al. (2019a), en su estudio sobre la experimentación y su integración en el proceso enseñanza aprendizaje de la física en la educación media, presenta una investigación fundamentada en una metodología de tipo mixto, pues en la misma participan componentes de tipo documental y de campo, además de cuali-cuantitativa pues usa elementos considerados exclusivamente cuantitativos pero apreciados desde una visión interpretativa, con la aplicación de un método sustentado en la hermenéutica dialéctica-interpretativa, donde la información fue obtenida implementando tres instrumentos tipo test que fueron aplicados a profesores, estudiantes y directivos de las Instituciones más representativas en cuanto a número de estudiantes, número de profesores, cantidad de secciones, historia y vivencias de las mismas en el marco del contexto socio cultural del estado Trujillo (Venezuela), durante el año 2018.

Así mismo en la investigación se usó como herramienta la entrevista semiestructurada con algunos de los participantes y las anotaciones en el trabajo de campo realizadas mediante la observación de la dinámica funcional y condiciones estructurales y de equipamiento de cada una de las Instituciones mencionadas, triangulando y validando la información aportada. El análisis e interpretación de la misma, llevó a configurar un panorama poco alentador relacionado a la asimilación de un conocimiento integral de parte de los estudiantes en la enseñanza de esta ciencia, la experimentación esencialmente está ausente de la práctica docente, por otra parte en la mayoría de las instituciones no existe un laboratorio en cuanto tal y en las que lo tienen, el tiempo destinado a la ejecución de actividades prácticas es destinado a otros asuntos, incluso los experimentos de tipo demostrativo son realmente escasos, concluyéndose que las prácticas instruccionales han permeado el currículo promoviendo con ello solamente el aprendizaje de tipo memorístico. Convirtiendo de esta manera una ciencia que intenta interpretar la realidad de nuestro entorno, en la memorización de algunas expresiones en la pretensión que manipuladas adecuadamente permiten resolver algunos problemas típicos, desconectados del contexto vivencial del estudiante. 
De igual manera Briceño et al (2019b) en su trabajo sobre la dimensión afectividad en la praxis cotidiana del docente de Física presentan una investigación que tuvo como objetivo lograr una estructura teórica consensuada sobre el rol de la dimensión afectividad en la praxis pedagógica cotidiana del docente de Física en la construcción de saberes en la Educación Universitaria (en particular el caso Venezuela). Centrada en procesos socioculturales y orientada bajo el paradigma cualitativo, sustentado ontológica y epistemológicamente en la fenomenología y la hermenéutica. La información se obtuvo mediante entrevista en profundidad a los protagonistas, representados por profesores universitarios seleccionados con el criterio denominado bola de nieve. Los fenómenos fueron estudiados según la perspectiva de los sujetos, considerando su marco referencial, sea en el contexto donde se desenvolvieron como agentes subjetivos biográficos, como en sus múltiples contingencias experimentadas, las cuales generaron actitudes y saberes.

La triangulación como contraste proporcionó credibilidad y validez al procedimiento. Surgió que la afectividad está presente en las acciones del docente, por tanto, las emociones representan elementos esenciales en la asimilación de saberes de la Física, es a través del sentir que el docente se hace ideas de la realidad e interactúa con el educando, por ello, no se pueden desligar del plano cognitivo del ser, en el proceso de enseñar contenidos de la Física. Se evidenció que la empatía, constituye el motor de las actividades en aula y una de las bases más sólidas sobre las que se fundamenta el bienestar de los docentes y la dialógica docente-estudiante, de consecuencia para comprender en toda su complejidad el acto de aprender en Física se requiere ir más allá de lo cognitivo.

En cuanto a lo que es la motivación, Santrock (2002), la define como "el conjunto de razones por las que las personas se comportan de las formas en que lo hacen. El comportamiento motivado es vigoroso, dirigido y sostenido" (p. 432), por su parte Ajello (2003) propone que la motivación debe ser percibida como la trama que sostiene el desarrollo de aquellas actividades que son significativas para la persona y en las que esta toma parte. De igual manera el ya mencionado autor indica que en el plano educativo, la motivación debe ser calificada como la disposición positiva para aprender y continuar haciéndolo de una forma autónoma.

Trechera (2005) explica que, etimológicamente, el término motivación procede del latín motus, que se relaciona con aquello que moviliza a la persona para ejecutar una actividad. En ese sentido, se puede definir la motivación como el proceso por el cual el sujeto se plantea un objetivo, utiliza los recursos adecuados y mantiene una determinada conducta, con el propósito de lograr una meta. Bisquerra (2000) por su parte propone que "la motivación es un constructo teórico-hipotético que designa un proceso complejo que causa la conducta. En la motivación intervienen múltiples variables (biológicas y adquiridas) que influyen en la activación, direccionalidad, intensidad y coordinación del comportamiento encaminado a lograr determinadas metas” (p. 165).

Por su parte Ramírez y Castillo (2020) indican que la motivación es un elemento fundamental e indispensable para que se alcancen las metas educativas durante el acto de enseñanza y aprendizaje de asignaturas como la matemática o la física. En contraposición Angeles (2020) señala que no existe una relación establecida y permanente entre la motivación y el aprendizaje significativo, por lo que este estudio ratificará uno de estos dos postulados.

Mendoza y Huamán (2020) exponen que la motivación académica es la actitud del aprendiz hacia lo que el estudiante percibe como la institución educativa. Los 
autores declaran que si la motivación es de buen nivel, los estudiantes activaran las conductas indicadas, de ese modo persistiendo y logrando el alcance de un determinado objetivo. Por tal razón es que el logro de un aprendizaje significativo considere la emoción, teniendo en cuenta a la motivación como parte de esa emoción. Los factores incidentes sobre la motivación hacia el aprendizaje son diversos, yendo desde lo perceptible hasta lo imperceptible. En ese sentido Lopez et al (2021) señalan que existe un efecto subyacente en la implementación de estrategias innovadoras para el aprendizaje, acogiendo aspectos situados y reconociendo la íntima relación entre afectividad y cognición. Igualmente Cáceres et al (2021) indican que el profesor es el agente con mejores herramientas, por lo tanto debe ser capaz de dar sentido y motivar a sus estudiantes para el alcance de los aprendizajes, por lo que al estudiar la motivación en el aprendizaje se debe tener en cuenta siempre a esta figura.

Los estudios ya mencionados respaldan la necesidad de desarrollar investigaciones en el área de aprendizaje de la física. La investigación que se presenta tiene como propósito fundamental conocer de qué manera inciden de los niveles motivacionales presentes en el aprendizaje de la física en estudiantes de los tres grados de BGU, en una unidad educativa particular de la ciudad de Guayaquil (Región Costa/Galápagos) en la Republica de Ecuador. En este estudio se pretende abordar y analizar cuestiones que generan una gran preocupación tanto a autoridades educativas como a instituciones de enseñanza, y por supuesto que a los docentes de física. Cuestiones como el aprendizaje de la física en un nivel escolar, la relevancia de la física como asignatura, las tensiones existentes entre la necesidad de que los alumnos aprendan física y la relevancia de la física como asignatura entre los aprendices son abordadas. Todo ello abordado desde una perspectiva de la realidad nacional ecuatoriana, que no solo busca contribuir con la reflexión desde el quehacer académico sino también con la mejoría de los procesos de enseñanza y aprendizaje de la física.

\section{Metodología}

\subsection{Enfoque y tipo de investigación}

El presente estudio por su naturaleza es cuantitativo, tipo descriptivo. En cuanto al diseño, se empleó un diseño no experimental transversal.

\subsection{Unidades de análisis}

La población se compone de los estudiantes activos en el sistema educativo ecuatoriano cursantes de alguno de los grados conducentes a obtener la titulación de bachillerato general unificado (primero, segundo y/o tercero) residentes en la ciudad de Guayaquil.

La muestra estuvo compuesta por 330 estudiantes activos de BGU, durante el año lectivo 2021-2022 de la región Costa/Galápagos de una institución educativa privada (particular). La selección de la muestra se llevó a cabo al emplear un muestreo intencional.

Todos los participantes al tiempo de la aplicación de los instrumentos estaban matriculados en la misma institución educativa. Con edades comprendidas entre los 14 y 18 años, distribuidos de la siguiente manera: 110 estudiantes cursantes de cada uno de los años de BGU, siendo 55 mujeres y 55 hombres. 


\subsection{Técnicas de recolección de datos}

Con el propósito de recolectar la información suministrada por los participantes se procedió con la aplicación de un instrumento, llamado "Test para mediar la actitud motivación hacia el aprendizaje de física”. Compuesto por 32 ítems presentados como proposiciones afirmativas, organizados en una escala Likert con valores de 1 a 5 puntos (5 puntos para Totalmente de acuerdo, 4 puntos para De acuerdo, 3 puntos para Ni de acuerdo, ni en desacuerdo, 2 puntos para En desacuerdo, 1 punto para Totalmente en desacuerdo). La puntuación total para el Test presentado por cada individuo se obtiene sumando los valores por ítems, con un valor máximo posible de 160 y un valor mínimo posible de 32 puntos.

El instrumento cuenta con una confiabilidad de 0,89 puntos de acuerdo al Alfa de Cronbanch, y la validez del mismo estriba en la correlación predicativa que los ítems tienen con categorías consideradas que miden motivación.

Los factores considerados en el instrumento se presentan a continuación:

- Interés en la física como asignatura, ítems 1, 2, 3, 4.

- Intensidad de la motivación, ítems 5, 6, 7, 8 .

- Actitud hacia el docente de física 9, 10, 11, 12.

- Actitud hacia el aprendizaje de física 13, 14, 15, 16.

- Ventajas a futuro que vienen por sobresalir en física 17, 18, 19, 20.

- Deseo de aprender física 21, 22, 23, 24.

- Actitud hacia el entorno escolar y los compañeros con los que se aprende física $25,26,27,28$.

- Orientación Instrumental 29, 30, 31, 32.

\subsection{Procesamiento y análisis de la información}

Previa selección y acuerdo voluntario de participación se procedió a verificar que cada uno de los estudiantes de la muestra cumpliese con las características necesarias para considerar su actuación bajo consentimiento informado (capacidad física y cognitiva de participar en la aplicación de un instrumento, voluntariedad de participación, información detallada sobre la realización del instrumento y fines de la participación y comprensión tanto del instrumento como de lo que se requiere de ellos como participantes). Al corroborarse este factor de participación, se procedió a compartir los enlaces contentivos con los participantes. El instrumento fue aplicado de forma asincrónica, empleando la herramienta virtual de "Google Forms" para cada instrumento.

Una vez obtenidos los datos en su totalidad, se procesaron utilizando "Paquete estadístico para las ciencias sociales” (SPSS), que emplea procesos matemáticos de la estadística descriptiva, para ello se usaron modelos de regresión, modelos avanzados de reducción de datos y de clasificación, así como análisis de tendencia y de conjunto, pruebas exactas y análisis de valores perdidos, por último, se empleó el software para ejecutar la validación de datos. Acto seguido, la información fue jerarquizada, organizándola y presentándola en tablas de frecuencia, y gráficos según su utilidad. El instrumento se aplicó durante el mes de junio del año 2021.

A fin de establecer los niveles de motivación respecto al aprendizaje de física se empleó una escala de medición ordinal, creada a partir de la distribución equitativa de los puntajes posibles entre cinco categorías determinantes de los niveles motivacionales, la distribución de los puntajes se organizó a partir de la consideración de los puntajes máximo y mínimo posibles. (Ver Tabla 1) 
Tabla 1. Escala para medir los niveles motivacionales en el aprendizaje de la física

\begin{tabular}{|c|c|}
\hline Nivel de Motivación & Puntaje \\
\hline Máximo (Muy motivado) & $134-160$ \\
\hline Alto (Altamente motivado) & $108-133$ \\
\hline Medio (Medianamente motivado) & $82-107$ \\
\hline Baja (Poco motivado) & $56-81$ \\
\hline Mínimo (Muy poco motivado) & $32-55$ \\
\hline
\end{tabular}

Fuente: Datos obtenidos a partir de la aplicación del instrumento. Briceño (2021)

\section{Resultados}

\subsection{Niveles motivacionales}

Una vez procesados los datos se asegura que los estudiantes presentan una media motivacional de 110 puntos, lo que quiere decir que se encuentran altamente motivados hacia el aprendizaje de la física (Ver Figura 1). Los estudiantes del primer año BGU participantes mostraron una media de 109 puntos, en la que las estudiantes mujeres una media motivacional de 108 puntos y los estudiantes hombres presentaron una media de 111 puntos. En el caso de los estudiantes del segundo año de BGU la media motivacional fue de 108 puntos, en la que las mujeres presentaron una media motivacional de 107 puntos y los hombres presentaron una media motivacional de 109. Finalmente en el caso de los estudiantes de tercer año de BGU la media motivacional fue de 112, para este grado las mujeres presentaron una media motivacional de 112 puntos y los hombres una media motivacional de 111 (Ver Figura 2)

Figura 1. Niveles motivacionales presentes en estudiantes de BGU

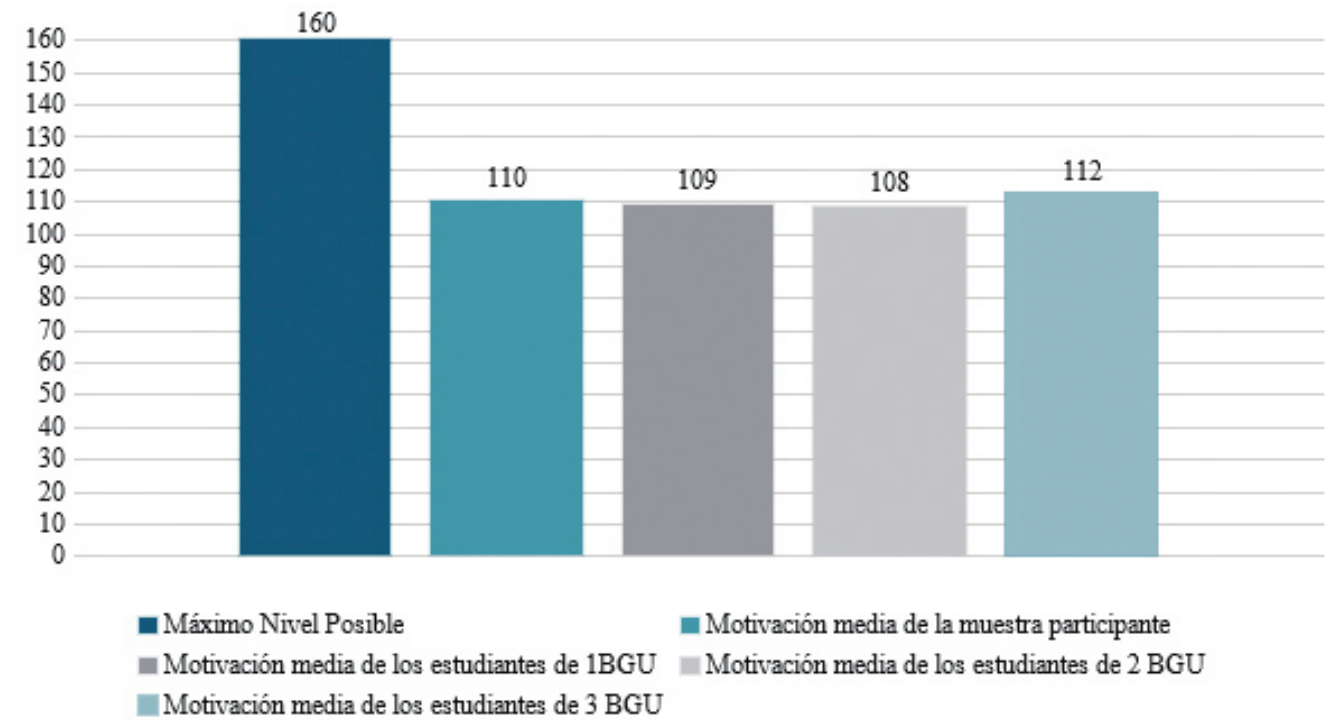

Fuente: Datos obtenidos a partir de la aplicación del instrumento. Briceño (2021) 
Figura 2. Niveles motivacionales por género presentes en estudiantes de BGU participantes

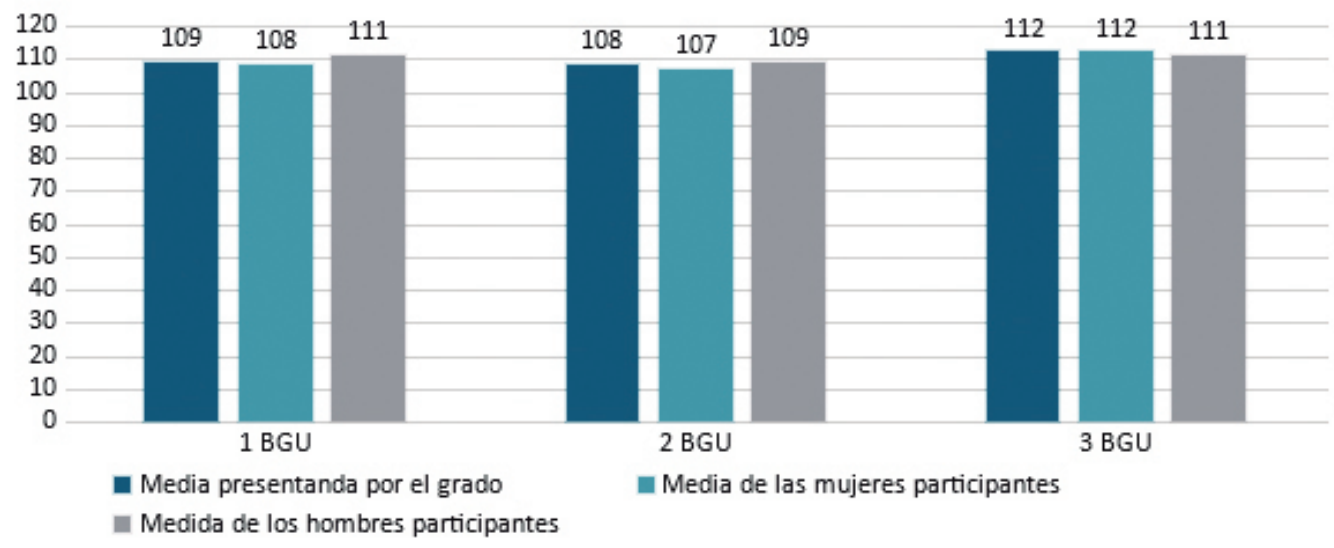

Fuente: Datos obtenidos a partir de la aplicación del instrumento. Briceño (2021)

\subsection{Factores motivacionales}

Con el propósito de identificar el impacto por factor sobre los niveles generales de motivación en el aprendizaje de la física en estudiantes de bachillerato, se presenta a continuación un análisis a partir de los porcentajes mayores obtenidos, según el grupo académico, es decir: primer, segundo y tercer año de BGU.

\subsubsection{Interés de la física como asignatura}

El $56 \%$ de los estudiantes de primer año de BGU afirmaron estar interesados en el aprendizaje de la física como asignatura, siendo que el $76 \%$ de los estudiantes expresó deseos de poder resolver los ejercicios de física con rapidez. Por su parte al tratarse de los estudiantes de segundo año de BGU el $60 \%$ de los estudiantes manifestó tener interés en la física como asignatura, con un $76 \%$ de los estudiantes expresando que desean poder resolver ejercicios de física con rapidez. Finalmente los estudiantes de tercer año de BGU dejan ver que el $66 \%$ tiene un alto interés en la física como asignatura, siendo que el $82 \%$ de los participantes de este grupo desean poder resolver ejercicios de física con rapidez.

\subsubsection{Intensidad de la motivación}

El $53 \%$ de los estudiantes de primer año de BGU demostró tener una motivación intensa al tratarse del aprendizaje de la física. El 68 \% de los estudiantes señaló poner mucha atención para tratar de aprender todo lo posible en la clase de física. Por su parte al tratarse de los estudiantes de segundo año de BGU, el $50 \%$ de ellos mostró una intensidad motivacional alta al tratarse del aprendizaje de física, con el $67 \%$ de los estudiantes que expresan poner mucha atención para lograr un aprendizaje significativo. Finalmente los estudiantes de tercer año de BGU dejan ver que el $57 \%$ tienen un alto nivel en la intensidad de su motivación, y en ese particular el $70 \%$ de los estudiantes expresa que pone mucha atención para tratar de aprender todo lo posible en clases de física. 


\subsubsection{Actitud hacia el docente de física}

El 67 \% de los estudiantes de primer año de BGU expresó tener una actitud positiva hacia como perciben al docente que les imparte física como asignatura. El 80 $\%$ de los estudiantes declaró que les agradaba su profesor de física y que les agradaba la forma como su profesor impartía la asignatura. Por su parte al tratarse de los estudiantes de segundo año de BGU, el $55 \%$ valora positivamente a su profesor y la forma como enseña la asignatura, al respecto el $85 \%$ de los estudiantes participantes señaló que les agrada mucho su profesor de física. Finalmente los estudiantes de tercer año de BGU dejan ver que el $81 \%$ de ellos tienen una actitud positiva hacia el profesor de física, y el $91 \%$ de los participantes expresan percibir con mucho agrado a su profesor de física.

\subsubsection{Actitud hacia el aprendizaje de física}

El $38 \%$ de los estudiantes de primer año de BGU demostró tener una actitud neutral hacia el aprendizaje de la física. Igualmente el $47 \%$ en este grupo de participantes señaló neutralidad en cuanto al disfrute y el gusto en el aprendizaje de física.

Por su parte al tratarse de los estudiantes de segundo año de BGU, el $45 \%$ de los participantes presentó una valoración positiva hacia el aprendizaje de la física, y el $52 \%$ de los estudiantes dijo tener deseos de aprender tanto de física como les fuese posible. Finalmente los estudiantes de tercer año de BGU dejan ver que el 50 $\%$ de ellos tienen una actitud positiva de la física como asignatura y la valoran como importante, siendo que el $61 \%$ de los participantes piensan que la clase de física es una parte muy importante del programa de estudio.

\subsubsection{Ventajas a futuro que da el saber física}

El $56 \%$ de los estudiantes de primer año de BGU perciben que el saber física es algo ventajoso a futuro para ellos, tal es que el $61 \%$ indicó que estudiar física es importante porque les permitirá sentirse cómodos cuando estén en las clases de la universidad. Por su parte al tratarse de los estudiantes de segundo año de BGU, el 57 $\%$ de los estudiantes señaló que el conocer de física representa una ventaja competitiva en el campo académico, el $61 \%$ dijo que el estudiar física es importante porque les permitiría entender y apreciar mejor otras asignaturas importantes. Finalmente los estudiantes de tercer año de BGU dejan ver que un $45 \%$ de ellos consideran que saber física representa una ventaja en su futuro, y el $50 \%$ de los participantes piensan que ese conocimiento les permitirá conocer mejor otros aspectos relacionados con otras asignaturas.

\subsubsection{Deseo de aprender física}

El $38 \%$ de los estudiantes de primer año de BGU señaló una actitud neutra en cuanto a su deseo de aprender física, y el 47 \% señaló no estar en acuerdo ni en desacuerdo respecto al aprender todo lo posible en cuanto a lo relacionado con los temas de la asignatura. Por su parte al tratarse de los estudiantes de segundo año de BGU, el $39 \%$ demostró un deseo fuerte de aprender física, sin embargo el $75 \%$ de los participantes señaló que les gustaría aprender física a la perfección. Finalmente los estudiantes de tercer año de BGU dejan ver que el $41 \%$ de ellos presenta una actitud neutral hacia el aprendizaje de física como asignatura. 


\subsubsection{Actitud hacia el entorno escolar y los compañeros con los que se aprende física}

El $44 \%$ de los estudiantes de primer año de BGU indicó una actitud neutral en relación con el entorno escolar y los compañeros con los que se aprende física, tal es que en la mayoría de los ítems relacionados con este factor los estudiantes seleccionaron la opción "Ni de acuerdo ni en desacuerdo". Por su parte al tratarse de los estudiantes de segundo año de BGU, el $46 \%$ de los estudiantes demostraron una valoración negativa hacia el entorno escolar y los compañeros con los que se aprende física, siendo que el $57 \%$ no se siente cómodos en el entorno, y desearía aprender física con otro grupo de estudiantes. Finalmente los estudiantes de tercer año de BGU dejan ver que una mayoría del $44 \%$ tiene una percepción neutral sobre el entono en el que aprenden física, y sus compañeros de clases.

\subsubsection{Orientación instrumental}

El $41 \%$ de los estudiantes de primer año de BGU demostró tener una alta orientación instrumental en el aprendizaje de física, sin embargo un $40 \%$ señaló tener una orientación instrumental media. Por su parte al tratarse de los estudiantes de segundo año de BGU, el $44 \%$ presentó una fuerte orientación instrumental hacia el aprendizaje de la física, en la que el $47 \%$ de los participantes piensa que estudiar física es importante porque se les respetará más si dominan esa asignatura. Finalmente los estudiantes de tercer año de BGU dejan ver que el $42 \%$ tiene una orientación instrumental media, tal es que el $48 \%$ de ellos no están seguros sobre que tanto les sirva o no el tener conocimientos de física.

\section{Conclusiones}

Los resultados obtenidos permiten declarar que efectivamente los estudiantes de BGU participantes se encuentran motivados hacia el aprendizaje de física, y con el puntaje obtenido se puede establecer que los estudiantes están altamente motivados en el aprendizaje de esta asignatura. Los estudiantes con la mayor motivación fueron los del tercer año de GBU, y los menos motivados fueron los estudiantes del segundo año de BGU, aunque es necesario mencionar que sólo están un punto por debajo de la media de los estudiantes del primer año de BGU, quienes presentaron una motivación semejante. En general los estudiantes hombres de este estudio están más motivados al aprendizaje de la física de lo que están las mujeres participantes en esta investigación, exceptuando el tercer año de BGU, en el que las mujeres tuvieron una motivación ligeramente por encima de la de los hombres. Estos datos pudiesen servir de referente a futuras investigaciones ya que por las características de la muestra y el tipo de investigación no se les puede considerar concluyentes ni definitivos.

En cuanto a los factores motivacionales y su incidencia individual sobre el nivel general de motivación se puede asegurar que todos impactan de manera directa y significativa sobre la forma como los estudiantes perciben el aprendizaje de la asignatura. Más de la mitad de ellos declaran tener un alto interés en lo concerniente al aprendizaje de la física como asignatura, y lo mismo sucede al evaluar la intensidad de la motivación y la forma como perciben a su docente.

Los estudiantes pertenecientes a la muestra en su mayoría (más de la mitad) presentan una actitud indiferente o negativa hacia el aprendizaje de física como asignatura. Los resultados demuestran que el deseo de aprender física es igualmente bajo. Más de la mitad de los estudiantes participantes no tienen una orientación instrumental positiva. Por otro lado, más de la mitad de los estudiantes demostraron 
no sentirse completamente cómodos con el entorno escolar y los compañeros con los que se aprende física. Pese a lo antes referido, más de la mitad reconoce que el tener un dominio académico de la física les va a garantizar una posición aventajada frente a situaciones y contextos académicos exigentes.

Estudios como el presentado permiten que los actores del proceso educativo analicen los niveles motivacionales incidentes en el aprendizaje de la física, así como el impacto que cada factor tiene sobre la motivación en cada estudiante, atendiendo a su grupo y contexto académico más que a su edad. El estudio sobre motivación y género en cuanto al aprendizaje presenta un campo fértil de estudio que merece ser expandido y analizado a profundidad, siendo que los datos reflejados no se presentan como definitivos. Se puede declarar que los estudiantes del estudio presentan un alto grado de motivación, y que cada uno de los factores estudiados, a saber: el interés de la física como asignatura, la intensidad de la motivación del estudiante, la actitud hacia el docente de física, la actitud hacia el aprendizaje de física, las ventajas a futuro que da el saber física, el deseo de aprender física y la actitud hacia el entorno escolar y los compañeros con los que se aprende física inciden de manera directa y contundente sobre los estudiantes de bachillerato.

\section{Referencias}

Ajello, A. M. (2003). La motivación para aprender. En C. Pontecorvo (Coord.), Manual de psicología de la educación (pp. 251-271). España: Popular.

Angeles Niquen, C. R. (2020). Motivación y el aprendizaje significativo de los estudiantes de educación superior del Centro de Asesoría San Marcos, Lima 2019.

Bisquerra, R. (2000). Educación emocional y bienestar. Barcelona: CISSPRAXIS.

Briceño, J., Rivas, Y., y Lobo, H. (2019a). La Experimentación y su Integración en el proceso Enseñanza Aprendizaje de la Física en la Educación Media. RELACult - Revista Latino-Americana De Estudos Em Cultura E Sociedade, 5(2). https://doi. org/10.23899/relacult.v5i2.1512

Briceño, J., Rivas, Y., y Lobo, H. (2019b). La Dimensión Afectividad en la Praxis Cotidiana del Docente de Física. REL ACult-Revista Latino-Americana De Estudos Em Cultura E Sociedade, 5(4). https://doi.org/10.23899/relacult.v5i4.1144

Cáceres, C., Muñoz, C., y Valenzuela, J. (2021). Responsabilidad personal docente y motivación escolar. Revista Electrónica Interuniversitaria de Formación del Profesorado, 24(1). https://doi.org/10.6018/reifop.402761

Luque Carcasi, J. H. (2017). Estilos de aprendizaje y su relación con el rendimiento escolar en Física, de los estudiantes del quinto de secundaria en la Institución Educativa N 17, VES-2016.

Mendoza Rubio, A. M., y Huamán Suárez, S. G. (2020). Importancia de la motivación en el aprendizaje de los estudiantes.

López Ocampo, N., Álzate López, L., Llano, M., y Domínguez Rojas, A. (2020). Práctica pedagógica y motivación desde el aprendizaje situado. Tesis Psicológica, 16(1), 1-29. https://doi.org/10.37511/tesis.v16n1a9

Ramírez, M. D. R. R., y Castillo, H. I. O. (2020). Funciones cognitivas y motivación en el aprendizaje de las matemáticas. Naturaleza y Tecnología, (2).

Santrock, J. (2002). Psicología de la educación. México: Mc Graw-Hill.

Trechera, J. L. (2005). Saber motivar: ¿El palo o la zanahoria? http://www.psicologiaonline.com/articulos/2005/motivacion.shtml 